\title{
Utilizing Maps to Identify Possible Missed Cases of Chagas Disease
}

Chagas disease is one of the world's most neglected health conditions. It is a leading cause of heart disease in the Americas, creating a greater burden than any other parasitic infection. In 2013, Chagas became a reportable condition in Texas. Between 2013-17, the Texas Department of State Health Services confirmed 124 human and 436 canine cases. In the United States, less than $1 \%$ of people with the disease receive a clinical diagnosis, mainly due to low provider awareness and poor diagnostics.

The study's objective was to identify possible missed diagnosis of Chagas in Texas utilizing mapping software and public health data. It was hypothesized that higher rates non-ischemic cardiac disease and cardiomyopathy would be concentrated in areas of higher populations of Hispanic populations. ArcView 10.6 software was used to generate maps showing confirmed cases of Chagas along with cardiac diagnostic specific codes of Inpatient Public Use Data. Confirmed cases were not homogenously dispersed due to more testing being done near urban areas and research centers. However, heart-related diagnosis and age/ethnicity demographics showed the possibility of missed diagnosis throughout the state, especially along the border region. Maps indicated that areas to focus screenings are in rural counties with a higher percentage of Hispanics and with younger heart-related disease. Diagnostics for Chagas disease cases occurring in North America are considered to be very inaccurate. The majority of diagnostics are developed in South America based on large prevalence studies using genotypes not commonly found in North America (types I and IV). Future research in the United States should 
focus on screenings in higher-risk populations locally, which allows developing better diagnostic tools to identify cases accurately. By having reliable and affordable diagnostics, health care providers may actually screen patients therefore providing us with a better understanding of the prevalence of the disease. 